\title{
Obstructive sleep apnea combined dyslipidemia render additive effect on increasing atherosclerotic cardiovascular diseases prevalence
}

Zhiyong Cao ${ }^{1,2}$, Ping Zhang ${ }^{3}$, Zhiqing $\mathrm{He}^{1}$, Jing Yang ${ }^{1}$, Chun Liang ${ }^{1}$, Yusheng Ren ${ }^{1}$ and Zonggui Wu ${ }^{1 *}$

\begin{abstract}
Background: Current study was designed to investigate the effects of obstructive sleep apnea (OSA) combined dyslipidemia on the prevalence of atherosclerotic cardiovascular diseases (ASCVD).

Methods: This was a cross-sectional study and subjects with documented dyslipidemia and without previous diagnosis of OSA were enrolled. Polysomnography was applied to evaluate apnea-hypopnea index (AHI). Based on AHI value, subjects were classified into four groups: without OSA, mild, moderate and severe OSA groups. Clinical characteristics and laboratory examination data were recorded. Relationship between AHI event and lipid profiles was analyzed, and logistic regression analysis was used to evaluate the effects of OSA combined dyslipidemia on ASCVD prevalence.

Results: Totally 248 subjects with dyslipidemia were enrolled. Compared to the other 3 groups, subjects with severe OSA were older, male predominant and had higher smoking rate. In addition, subjects with severe OSA had higher body mass index, waist-hip ratio, blood pressure, and higher rates of overweight and obesity. Serum levels of fasting plasma glucose, glycated hemoglobin, LDL-C and CRP were all significantly higher. ASCVD prevalence was considerably higher in subjects with severe OSA. AHI event in the severe OSA group was up to $35.4 \pm 5.1$ events per hour which was significantly higher than the other groups $(P<0.05$ for trend). Pearson correlation analysis showed that only LDL-C was positively correlated with AHI events $(r=0.685, P<0.05)$. Logistic regression analysis revealed that in unadjusted model, compared to dyslipidemia plus no-OSA group (reference group), OSA enhanced ASCVD risk in subjects with dyslipidemia, regardless of OSA severity. After extensively adjusted for confounding variables, the odds of dyslipidemia plus mild-OSA was reduced to insignificance. While the effects of moderate- and severe-OSA on promoting ASCVD risk in subjects with dyslipidemia remained significant, with severe-OSA most prominent (odds ratio: 1.52, 95 \% confidence interval: 1.13-2.02).
\end{abstract}

Conclusion: OSA combined dyslipidemia conferred additive adverse effects on cardiovascular system, with severe-OSA most prominent.

Keywords: Dyslipidemia, Obstructive sleep apnea, Atherosclerotic cardiovascular diseases

\footnotetext{
* Correspondence: wu_zonggui@yeah.net

Cao zhiyong and Zhang Ping are co-first authors.

'Shanghai Changzheng Hospital, Second Military Medical University,

Shanghai 200003, China

Full list of author information is available at the end of the article
}

\section{Ciomed Central}

(c) 2016 The Author(s). Open Access This article is distributed under the terms of the Creative Commons Attribution 4.0 International License (http://creativecommons.org/licenses/by/4.0/), which permits unrestricted use, distribution, and reproduction in any medium, provided you give appropriate credit to the original author(s) and the source, provide a link to the Creative Commons license, and indicate if changes were made. The Creative Commons Public Domain Dedication waiver (http://creativecommons.org/publicdomain/zero/1.0/) applies to the data made available in this article, unless otherwise stated. 


\section{Background}

Obstructive sleep apnea (OSA) which is featured by intermittent hypoxemia during sleep is associated with atherosclerotic cardiovascular diseases (ASCVD) as revealed by previous epidemiological researches [1-3]. Owing to the increased pandemic of obesity $[4,5]$, a major risk factor of OSA, the incidence and prevalence of OSA is increased dramatically in recent decades [6]. Therefore, it is clinically relevant and important to screen, diagnose and treat OSA timely so as to reduce the ASCVD risk.

Dyslipidemia, as mainly defined by increased serum total cholesterol (TC) and low density lipoprotein cholesterol (LDL-C) levels, is another major risk of ASCVD. And a substantial amount of epidemiological and interventional researches have consistently demonstrated a linear relationship between serum LDL-C level and incident ASCVD [7]. In brief, LDL-C elevation is largely associated with increased consumption of saturated fat, which could also lead to central adiposity and obesity [8]. Therefore, it is conceivable that obesity may play overlapped roles between OSA and dyslipidemia, and OSA plus dyslipidemia may confer additive adverse effects on cardiovascular system. However, the evidence is lacking.

In our present cross-sectional research, we initially investigated the relationship between index of OSA and serum lipid profiles, and then evaluated the effects of OSA plus dyslipidemia on the prevalence of ASCVD. We hoped that information from our current study would broaden our understanding about the effects of OSA plus dyslipidemia on cardiovascular system, and additionally provide basics and insights for future clinical studies in managing OSA and dyslipidemia effectively so as to further reduce ASCVD risk.

\section{Methods}

\section{Studied subjects enrollment}

Present study was approved by the ethic committee of Shanghai Changzheng Hospital. Subjects were enrolled after informed consent was obtained, and the time of enrollment was from October of 2014 to October of 2015. All subjects had not been previously diagnosed as OSA, but were diagnosed as dyslipidemia according to selfreport, or treatment with statins, or increased serum TC and/or LDL-C level.

\section{Clinical characteristics and laboratory data collection}

Clinical characteristics including age, gender, smoking status, height and weight for calculating body mass index (BMI), waist and hip circumference for calculating waisthip ratio, systolic/diastolic blood pressure (SBP/DBP) and heart rate (HR) at rest were recorded in electronic case report form. ASCVD included previously diagnosed as coronary heart disease (CHD), ischemic stroke and peripheral artery disease (PAD) based on clinical symptoms plus objective evidence such as coronary angiography, computer tomography scan and Doppler ultrasound. Medical history and medicine usages were also collected. Laboratory data included fasting lipid profiles and plasma glucose (FPG), glycated hemoglobin (HbA1c) and C-reactive protein (CRP) were collected and double-checked by two working staffs.

\section{OSA diagnosis with polysomnography}

All enrolled subjects were underwent attended polysomnography and based on apnea-hypopnea index (AHI), those with $\mathrm{AHI} \geq 5$ events per hour were diagnosed as OSA [9]. In brief, AHI with 5-14.9 was defined as mild, 15-29.9 moderate and $\geq 30$ severe, and less than 5 was without OSA.

\section{Statistical analysis}

Continuous variables are presented with mean and SD and categorical variables are presented with the number and percentages. Statistical significance of differences is analyzed using one-way ANOVA or Mann-Whitney U test for continuous variables and the chi-square or Fisher exact test for categorical variables. Pearson correlation analysis was performed to evaluate the relationship between AHI and lipid profiles. Logistic regression analysis was applied to calculate odds ratio (OD) and associated $95 \%$ confidence intervals (CI) of OSA plus dyslipidemia on ASCVD prevalence. Statistical analysis is computed using SPSS 16.0 (SPSS Inc, Chicago, IL). All of the statistical tests were twosided and considered statistically significant if $P<0.05$.

\section{Results}

\section{Comparisons of clinical characteristics}

Totally, 248 subjects with dyslipidemia were enrolled, and according to the severity of OSA, all subjects were classified into four groups as presented in Table 1. Compared to the other three groups, subjects in the severe OSA group were older, male predominant and had higher rate of smoking $(P<0.05$ for trend). Furthermore, subjects with severe OSA appeared to at correspondingly higher prevalence of OSA risk factors as reflected by higher BMI, waist-hip ratio, blood pressure, and higher rates of overweight and obesity $(P<0.05$ for trend). With respect to laboratory examination, serum levels of FPG, HbA1c, LDL-C and CRP were all significantly higher in severe OSA group $(P<0.05$ for trend). ASCVD prevalence including $\mathrm{CHD}$, ischemic stroke and PAD was also considerably higher in subjects with severe OSA, and higher rates of medicines application might reflect the higher ASCVD risk $(P<0.05$ for trend). All these data revealed a linear relationship between OSA severity and ASCVD risk. AHI event in the severe OSA group was 
Table 1 Comparisons of clinical characteristics

\begin{tabular}{|c|c|c|c|c|}
\hline Variables & Without OSA & Mild & Moderate & Severe \\
\hline $\mathrm{N}$ & 56 & 74 & 65 & 53 \\
\hline Age (years) & $49.4 \pm 11.6$ & $52.2 \pm 13.6$ & $53.3 \pm 12.2$ & $55.3 \pm 13.1^{*}$ \\
\hline Male, $n(\%)$ & $30(53.6)$ & $41(55.4)$ & $35(53.8)$ & $31(58.5)^{*}$ \\
\hline Smoking, $n(\%)$ & $24(42.9)$ & $37(50.0)$ & $32(49.2)$ & $28(52.8)^{*}$ \\
\hline $\mathrm{BMI}\left(\mathrm{Kg} / \mathrm{m}^{2}\right)$ & $24.2 \pm 2.7$ & $25.5 \pm 3.5$ & $25.8 \pm 4.6$ & $26.3 \pm 3.9^{*}$ \\
\hline Overweight, $n$ (\%) & 10(17.9) & $30(40.5)$ & $31(47.7)$ & $27(50.9)^{*}$ \\
\hline Obesity, n (\%) & $0(0)$ & $0(0)$ & $3(4.6)$ & $8(15.1)^{*}$ \\
\hline Waist-hip ratio & $0.90 \pm 0.03$ & $0.92 \pm 0.05$ & $0.94 \pm 0.04$ & $0.96 \pm 0.03^{*}$ \\
\hline $\mathrm{SBP}(\mathrm{mm} \mathrm{Hg})$ & $132.5 \pm 10.2$ & $131.7 \pm 12.3$ & $135.8 \pm 11.6$ & $138.2 \pm 13.4^{*}$ \\
\hline DBP (mm Hg) & $73.6 \pm 8.8$ & $73.2 \pm 10.5$ & $77.3 \pm 9.1$ & $81.2 \pm 10.6^{*}$ \\
\hline HR (bpm) & $74.2 \pm 6.6$ & $80.4 \pm 9.2$ & $88.4 \pm 9.2$ & $93.6 \pm 11.2^{*}$ \\
\hline FPG (mmol/L) & $6.0 \pm 1.1$ & $6.2 \pm 1.7$ & $6.4 \pm 1.5$ & $6.7 \pm 1.6^{*}$ \\
\hline $\mathrm{HbA1c}(\%)$ & $6.0 \pm 1.3$ & $6.3 \pm 1.3$ & $6.6 \pm 1.3$ & $6.8 \pm 1.5^{*}$ \\
\hline TC (mmol/L) & $5.3 \pm 0.5$ & $5.3 \pm 0.4$ & $5.4 \pm 0.5$ & $5.5 \pm 0.6$ \\
\hline TG (mmol/L) & $1.3 \pm 0.4$ & $1.4 \pm 0.4$ & $1.4 \pm 0.3$ & $1.5 \pm 0.4$ \\
\hline LDL-C (mmol/L) & $3.1 \pm 0.4$ & $3.3 \pm 0.4$ & $3.3 \pm 0.3$ & $3.5 \pm 0.5^{*}$ \\
\hline $\mathrm{HDL}-\mathrm{C}(\mathrm{mmol} / \mathrm{L})$ & $1.0 \pm 0.3$ & $0.9 \pm 0.3$ & $0.8 \pm 0.3$ & $0.8 \pm 0.4$ \\
\hline CRP (mg/L) & $9.2 \pm 1.2$ & $10.8 \pm 2.4$ & $12.7 \pm 2.6$ & $14.2 \pm 3.3^{*}$ \\
\hline CHD, n (\%) & $6(10.7)$ & $10(13.5)$ & $11(16.9)$ & $10(18.9)^{*}$ \\
\hline Ischemic stroke, $n(\%)$ & $3(5.4)$ & $4(5.4)$ & $6(9.2)$ & $6(11.3)^{*}$ \\
\hline PAD, n (\%) & $3(5.4)$ & $3(4.1)$ & $4(6.2)$ & $5(9.4)^{*}$ \\
\hline Anti-platelet, $n(\%)$ & $22(39.3)$ & $34(45.9)$ & $32(49.2)$ & $28(52.8)^{*}$ \\
\hline ACEI/ARB, $n(\%)$ & $26(46.4)$ & $36(48.6)$ & $34(52.3)$ & $29(54.7)^{*}$ \\
\hline Statins, $n(\%)$ & 29(51.8) & $37(50.0)$ & $35(53.8)$ & $31(58.5)^{*}$ \\
\hline AHI (events/h) & $3.2 \pm 1.3$ & $12.6 \pm 2.3$ & $21.2 \pm 3.3$ & $35.4 \pm 5.1^{*}$ \\
\hline
\end{tabular}

Denote: ${ }^{*} P<0.05$ versus other groups; bpm beat per minute, $T G$ triglyceride, HDL-C high density lipoprotein cholesterol, $A C E l$ angiotensin converting enzyme inhibitor, $A R B$ angiotensin receptor blocker

up to $35.4 \pm 5.1$ events per hour which was significantly higher than the other groups $(P<0.05$ for trend).

\section{Relationship between lipid profiles and AHI events}

Pearson correlation analysis was applied to evaluate the relationship between serum TC, TG, LDL-C and HDL-C levels and AHI events. As shown in Table 2 and Fig. 1 that only LDL-C were positively correlated with AHI events, with correlation coefficient was $0.685(P<0.05)$.

Table 2 Relationship between lipid profiles and AHI events

\begin{tabular}{lcc}
\hline Variables & Correlation coefficient & $P$ value \\
\hline TC & 0.342 & 0.074 \\
TG & 0.228 & 0.107 \\
LDL-C & 0.685 & 0.002 \\
HDL-C & -0.236 & 0.089 \\
\hline
\end{tabular}

And the other lipid indices were not significantly correlated with AHI events.

\section{Effects of OSA plus dyslipidemia on ASCVD prevalence} Logistic regression analysis was used to analyze the effects of OSA plus dyslipidemia on ASCVD prevalence. As shown in Table 3, in the unadjusted model, compared to the dyslipidemia plus no-OSA group (reference group), OSA enhanced ASCVD risk in subjects with dyslipidemia, regardless of OSA severity. After adjusted for age, gender, BMI and waist-hip ratio (model 1), the odds of dyslipidemia plus mild-OSA on increasing ASCVD prevalence was reduced to insignificance. While the effects of moderate-OSA and severe-OSA on promoting ASCVD risk in subjects with dyslipidemia remained significant. Additional adjusted for SBP, FPG, LDL-C, CRP and statins (model 2), the effect of moderate-OSA and severe-OSA on enhancing ASCVD risk was still significant, with severe-OSA most prominent (OR: 1.52, 95 \% CI: 1.13-2.02). 

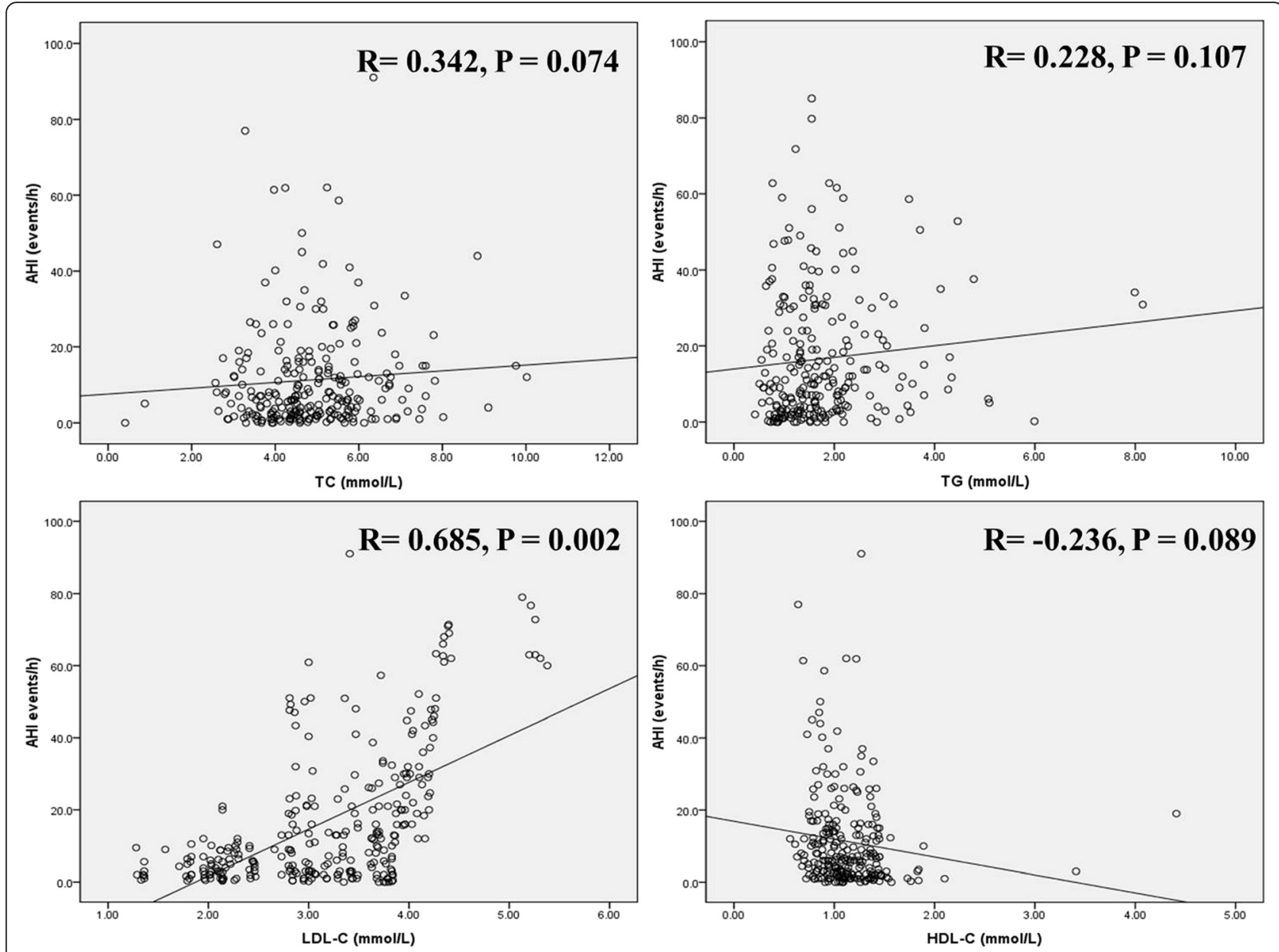

Fig. 1 Relationship between lipid profiles and AHI events

\section{Discussion}

Dyslipidemia and OSA both are major risk factors of ASCVD, and theoretically these two co-morbidities may confer additional risk on ASCVD. Data from our current study support this concept. We observe that there is a linear relationship between OSA severity and cardiovascular risk profiles in subjects with documented dyslipidemia; secondly, there is a positive correlation between serum LDL-C level and AHI events; thirdly, logistic regression analysis shows that dyslipidemia plus OSA confer additive effect on increasing ASCVD prevalence, with severe-OSA most prominent.
With increasing pandemic of obesity, OSA has now become more prevalent and imposes great public health burden owing to its close association with ASCVD. Mechanistically, through high-frequency intermittent hypoxemia and arousals, OSA elicits systemic inflammation, oxidative stress, sympathetic nerve activation and endothelial dysfunction [10-12]. All these pathological changes are detrimental to cardiovascular system. Indeed, a substantial amount of epidemiological studies have showed that OSA is independently associated with cardiovascular events. For example, Gottlieb DJ et al. reported that OSA was a significant predictor of incident

Table 3 Effects of OSA plus dyslipidemia on ASCVD prevalence

\begin{tabular}{lllll}
\hline Model & Dyslipidemia + no-OSA & Dyslipidemia + mild-OSA & Dyslipidemia + moderate-OSA & Dyslipidemia + severe-OSA \\
\hline Unadjusted & 1 & $1.54(1.16-2.07)^{*}$ & $1.77(1.42-2.24)^{*}$ & $1.96(1.35-2.62)^{*}$ \\
Mode I & 1 & $1.27(0.89-1.84)$ & $1.36(1.07-1.86)^{*}$ & $1.84(1.29-2.33)^{*}$ \\
Mode 2 & 1 & $1.09(0.81-1.77)$ & $1.11(0.92-1.75)^{*}$ & $1.52(1.13-2.02)^{*}$ \\
\hline
\end{tabular}


CHD and heart failure in community-dwelling middleaged and older men [13]. In another epidemiological study, Punjabi NM and colleagues had demonstrated that OSA was associated with all-cause mortality especially that ascribed to CHD [14]. Moreover, Redline S and coworkers also had revealed that there was a strong association between AHI event and ischemic stroke in community-dwelling men with mild to moderate sleep apnea [15]. All these evidence collectively supports the notion that OSA increases risk of ASCVD.

Other than OSA, obesity is also closely associated with dyslipidemia. And the adverse effects of dyslipidemia, especially in subjects with obesity, on cardiovascular system have been consistently demonstrated in previous clinical researches [16, 17]. Nonetheless, whether OSA could enhance the detrimental effects of dyslipidemia on cardiovascular system is not fully clear yet. We therefore conducted an observational research with cross-sectional design to evaluate the effects of OSA plus dyslipidemia on ASCVD prevalence. In brief, subjects with documented dyslipidemia were enrolled and classified into four groups based on OSA degree in order to evaluate the variable effects of different OSA degree plus dyslipidemia on ASCVD prevalence. In unadjusted model, OSA, regardless of severity, rendered additional risk of ASCVD in subjects with documented dyslipidemia. Nevertheless, after extensively adjusted for potential confounding variables, only moderate-OSA and severe-OSA still had significant effects on increasing odds of ASCVD in subjects with dyslipidemia. To our best knowledge, the following aspects might partially explain these findings. On the one hand, the effects of mild-OSA which was reduced to insignificant might be due to the modest effect of OSA on cardiovascular system. Some previous epidemiological studies showed that only moderate or severe OSA had independent effects on cardiovascular system, which might support this speculation. On the other hand, it was reported that highfrequency intermittent hypoxemia contributed to dyslipidemia [18]. Therefore, severe OSA might further elevate serum LDL-C level and exaggerate the adverse effects related to dyslipidemia. Data from between-group comparison also supported this speculated as reflected by significantly higher serum LDL-C level in severe-OSA group compared to other groups. Last but not the least, since both OSA and dyslipidemia are associated with systemic inflammation as reflected by increased serum CRP level $[19,20]$. And it had been demonstrated that increased serum CRP level is associated with increased risk of ASCVD [21]. Therefore, through concurrently increasing serum CRP level, dyslipidemia plus OSA might enhance ASCVD risk in an OSA-severity dependant manner.

There were some limitations of current study meriting address here. Firstly, the nature of cross-sectional design could not allow us to draw causal relationship between OSA plus dyslipidemia and ASCVD prevalence. Secondly, relative small sample size might not allow us to identify other potential differences between groups. Thirdly, despite extensively adjusted for potential confounding variables, the unrecognized biases regarding our findings still could not be ruled out.

\section{Conclusion}

Our preliminary research showed that OSA increased ASCVD prevalence in subjects with documented dyslipidemia, and OSA plus dyslipidemia conferred additive adverse effects on cardiovascular system, with severe-OSA most prominent. In the future study, it is warranted to investigate whether improve OSA could reduce ASCVD risk associated with dyslipidemia.

\section{Acknowledgements}

We appreciate very much for the kindly help from Dr. Yan Liu.

Funding

The study is supported by NSFC $(81130065,81072981)$.

\section{Authors' contribution}

$C Z, Z P, H Z, Y J$ and LC performed this study, RY performed statistical analyses, and WZ designed and wrote this article. All authors read and approved the final manuscript.

\section{Competing interests}

The authors declare that they have no competing interests.

\section{Author details}

${ }^{1}$ Shanghai Changzheng Hospital, Second Military Medical University, Shanghai 200003, China. 'Branch of No. 411 Hospital, PLA, Shanghai 200003, China. ${ }^{3}$ Shanghai Changhai Hospital, Second Military Medical University, Shanghai 200003, China.

Received: 15 April 2016 Accepted: 17 May 2016

Published online: 26 May 2016

\section{References}

1. Shahar E, Whitney CW, Redline S, et al. Sleep-disordered breathing and cardiovascular disease: cross-sectional results of the sleep heart health study. Am J Respir Crit Care Med. 2001;163(1):19-25.

2. Bassetti CL, Milanova M, Gugger M. Sleep-disordered breathing and acute ischemic stroke: diagnosis, risk factors, treatment, evolution, and long-term clinical outcome. Stroke. 2006;37(4):967-72.

3. Wattanakit K, Boland L, Punjabi NM, Shahar E. Relation of sleep-disordered breathing to carotid plaque and intima-media thickness. Atherosclerosis. 2008;197(1):125-31.

4. Peppard PE, Young T, Palta M, Dempsey J, Skatrud J. Longitudinal study of moderate weight change and sleep-disordered breathing. JAMA. 2000; 284(23):3015-21.

5. Young T, Skatrud J, Peppard PE. Risk factors for obstructive sleep apnea in adults. JAMA. 2004;291(16):2013-6.

6. Worsnop CJ, Naughton MT, Barter CE, Morgan TO, Anderson Al, Pierce RJ. The prevalence of obstructive sleep apnea in hypertensives. Am J Respir Crit Care Med. 1998:157(1):111-5.

7. Boekholdt SM, Hovingh GK, Mora S, et al. Very low levels of atherogenic lipoproteins and the risk for cardiovascular events: a meta-analysis of statin trials. J Am Coll Cardiol. 2014;64(5):485-94.

8. Stefanutti C, Labbadia G, Athyros VG. Hypertriglyceridaemia, postprandial lipaemia and non-HDL cholesterol. Curr Pharm Des. 2014;20(40):6238-48.

9. Sleep-related breathing disorders in adults: recommendations for syndrome definition and measurement techniques in clinical research. The Report of an American Academy of Sleep Medicine Task Force. Sleep. 1999; 22(5): 667-89. 
10. Somers VK, Dyken ME, Clary MP, Abboud FM. Sympathetic neural mechanisms in obstructive sleep apnea. J Clin Invest. 1995;96(4):1897-904.

11. Ryan S, Taylor CT, McNicholas WT. Selective activation of inflammatory pathways by intermittent hypoxia in obstructive sleep apnea syndrome. Circulation. 2005;112(17):2660-7.

12. Kato M, Roberts-Thomson P, Phillips BG, et al. Impairment of endotheliumdependent vasodilation of resistance vessels in patients with obstructive sleep apnea. Circulation. 2000;102(21):2607-10.

13. Gottlieb DJ, Yenokyan G, Newman AB, et al. Prospective study of obstructive sleep apnea and incident coronary heart disease and heart failure: the sleep heart health study. Circulation. 2010;122(4):352-60.

14. Punjabi NM, Caffo BS, Goodwin JL, et al. Sleep-disordered breathing and mortality: a prospective cohort study. PLoS Med. 2009;6(8):e1000132.

15. Redline S, Yenokyan G, Gottlieb DJ, et al. Obstructive sleep apnea-hypopnea and incident stroke: the sleep heart health study. Am J Respir Crit Care Med. 2010;182(2):269-77.

16. Caglayan E, Blaschke F, Takata Y, Hsueh WA. Metabolic syndromeinterdependence of the cardiovascular and metabolic pathways. Curr Opin Pharmacol. 2005;5(2):135-42.

17. Berryman CE, Grieger JA, West SG, et al. Acute consumption of walnuts and walnut components differentially affect postprandial lipemia, endothelial function, oxidative stress, and cholesterol efflux in humans with mild hypercholesterolemia. J Nutr. 2013;143(6):788-94.

18. Drager LF, Jun J, Polotsky VY. Obstructive sleep apnea and dyslipidemia: implications for atherosclerosis. Curr Opin Endocrinol Diabetes Obes. 2010 17(2):161-5.

19. Punjabi NM, Beamer BA. C-reactive protein is associated with sleep disordered breathing independent of adiposity. Sleep. 2007;30(1):29-34

20. Dallmeier D, Koenig W. Strategies for vascular disease prevention: the role of lipids and related markers including apolipoproteins, low-density lipoproteins (LDL)-particle size, high sensitivity C-reactive protein (hs-CRP), lipoprotein-associated phospholipase A2 (Lp-PLA(2)) and lipoprotein(a) (Lp(a)). Best Pract Res Clin Endocrinol Metab. 2014;28(3):281-94.

21. Li JJ, Fang CH. C-reactive protein is not only an inflammatory marker but also a direct cause of cardiovascular diseases. Med Hypotheses. 2004;62(4):499-506.

\section{Submit your next manuscript to BioMed Central and we will help you at every step:}

- We accept pre-submission inquiries

- Our selector tool helps you to find the most relevant journal

- We provide round the clock customer support

- Convenient online submission

- Thorough peer review

- Inclusion in PubMed and all major indexing services

- Maximum visibility for your research

Submit your manuscript at www biomedcentral.com/submit

) Biomed Central 